\title{
Surgical and Functional Outcomes After Thoracic Outlet Syndrome Decompression via Supraclavicular Approach: A 10-Year Single Centre Experience
}

\author{
Jakub Kaczynski ${ }^{\mathrm{a}, \mathrm{b}}$, Louis Fligelstone ${ }^{\mathrm{a}}$
}

\begin{abstract}
Background: Thoracic outlet syndrome (TOS) results from compression of the neurovascular structures in the region of thoracic outlet. Treatment depends on the variant (venous, arterial, neurological or any combination of these), severity of symptoms and response to the conservative management. The aim was to assess surgical and functional outcomes after surgical decompression using the Disabilities of the Arm, Shoulder and Hand (DASH) questionnaire.
\end{abstract}

Methods: A retrospective review of all TOS cases operated in our department between 2002 and 2012. Collected data provided information on patients' demographics, TOS type, operative findings, complications and follow-up. The pre and post operative DASH questionnaire scores were recorded.

Results: Forty four patients (88\%) completed pre and post operative DASH questionnaire. Mean age was $42 \pm 10.78$ years (range $16-60)$ and 37 were women. Twenty seven patients had the combination of neurological and vascular symptoms. The reminder of patients presented with venous TOS (vTOS, $\mathrm{n}=5$ ), arterial TOS (aTOS, $\mathrm{n}=7$ ) and 3 cases with venous-arterial TOS. There were 40 elective and 4 emergency operations. All operations were performed under the general anaesthesia and via supraclavicular approach. Decompressive procedures consisted of an excision of the cervical $(n=11)$ or first rib $(n=23)$, combined cervical and first rib excision $(\mathrm{n}=10)$, scalenectomy $(\mathrm{n}=42)$ and division of soft tissue $(\mathrm{n}=24)$. Post operative complications included wound infection (n $=1)$, phrenic nerve traction injury $(\mathrm{n}=2)$ and pleural entries $(\mathrm{n}=$ 12). There were no brachial plexus or vascular injuries. Mean follow up was $11.7 \pm 15.4$ months. Mean DASH questionnaire scores improved post operatively across all TOS groups. When DASH

Manuscript accepted for publication March 12, 2013

\footnotetext{
aABM University Health Board, Vascular Surgery Department, Morriston Hospital, Swansea, SA6 6NL, United Kingdom

${ }^{\mathrm{b}}$ Corresponding author: Jakub Kaczynski, ABM University Health Board, Vascular Surgery Department, Morriston Hospital, Swansea, SA6 6NL, United Kingdom. Email: Jakub.Kaczynski@hotmail.co.uk
}

doi: http://dx.doi.org/10.4021/jcs152w scores were analysed according to the TOS type, the most marked post operative improvement was noted in aTOS with mean delta change of $36.99 \pm 22.72$, followed by vTOS $(26.22 \pm 16.89)$ and nTOS $(18.64 \pm 21.07)$. These findings were statistically significant with $\mathrm{P}<0.05$. Thirty nine $(78 \%)$ patients reported the return of upper limb function. Only 5 patients (nTOS) reported worsening of the limb function.

Conclusions: Careful selection of patients with meticulous surgical technique can produce excellent surgical and functional outcomes in all TOS types.

Keywords: Thoracic outlet syndrome; Supraclavicular approach; DASH; Surgical outcomes

\section{Introduction}

Thoracic outlet syndrome (TOS) is one of the most challenging conditions that vascular surgeons may deal with. This reflects the complexity of the condition and the overlap of symptoms that cross the specialties of orthopaedics, neurosurgery, chronic pain and vascular disease. TOS affects less than $1 \%$ of population and is symptomatic in $60 \%$ of cases [1]. The condition describes the combination of symptoms,

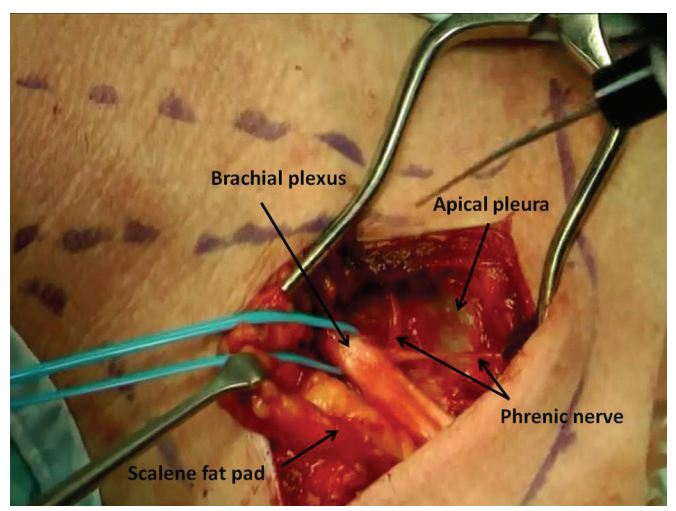

Figure 1. Thoracic outlet decompression via a supraclavicular approach. 


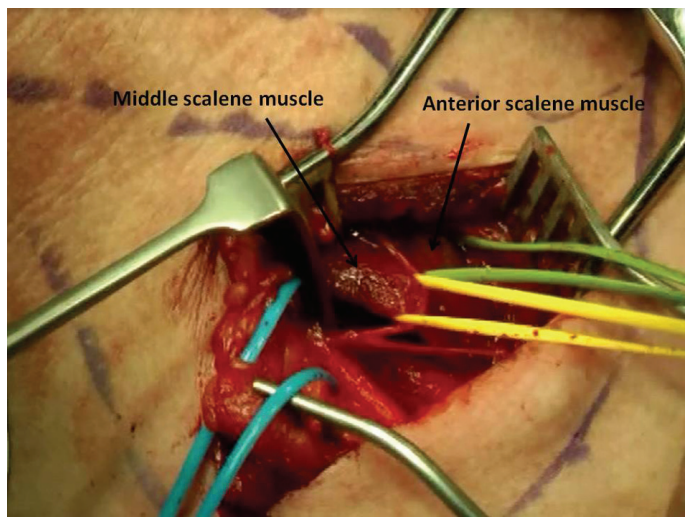

Figure 2. Hypertrophic scalene muscles.

which result from the compression of the neurovascular bundle in the region of the thoracic outlet $[2,3]$. The compression can be caused by the cervical ribs, elongated transverse C7 processes, bifid first rib, congenital fibro-muscular bands, hypertrophied scalene muscles and neck trauma $[4,5]$.

The scalene triangle is the most important anatomic space within the thoracic outlet. Its boundaries are formed by the anterior and middle scalene muscles, which comprise the sides of scalene triangle with the first rib forming the base of the triangle [5]. This relatively small space accommodates the subclavian artery (SCA), vein (SCV) and nerves of brachial plexus (Fig. 1, 2) [5, 6]. Therefore, produced symptoms depend on which structures are compressed. As a result, TOS is divided into three distinct subtypes, neurogenic (nTOS), venous (vTOS) and arterial (aTOS) [7, 8]. However, any combinations of these subtypes are possible. The most common one is nTOS (95\%), followed by vTOS (2-4\%) and aTOS (1\%) [1]. Neurogenic TOS is often subdivided further into the true and disputed forms, with the latter being most frequently encountered $[3,8]$.

Treatment algorithm depends on the type, severity of symptoms and response to conservative management (physiotherapy) $[7,9]$. Patients who fail the conservative treatment can benefit from surgical decompression of thoracic outlet by an excision of the cervical or first rib and scalenectomy with or without a revascularisation procedure $[2,6,10]$. The main goal of the treatment is to relieve the compression and most importantly to halt the progression of symptoms, which can lead to severe and long-term disability $[4,11]$. The literature quotes a failure rate following surgery between $7 \%$ and even $40 \%$ that may be associated with major complications $[1,9,12]$. For that reason, we feel it is necessary to describe our experience the surgical and functional outcomes following supraclavicular TOS decompression.

\section{Materials and Methods}

A retrospective review of prospectively collected data was performed of all patients who underwent surgical TOS decompression and completed Disabilities of the Arm, Shoulder and Hand (DASH) questionnaires during the 10-year period from 2002 to 2012 at our Vascular Surgery Department (ABM University Heath Board, Morriston Hospital, Swansea, United Kingdom). Collected data provided information on patients' demographics, TOS type, operative findings, complications and follow-up. Also pre and post operative DASH questionnaire scores were recorded.

We have used the DASH questionnaire which has been developed by the American Academy of Orthopaedic Surgeons (http:// www.dash.iwh.on.ca/) as a measure of the functional status and disability after surgery [13]. Several studies validated the questionnaire as an objective tool in evaluating post operative outcomes [7]. DASH question-

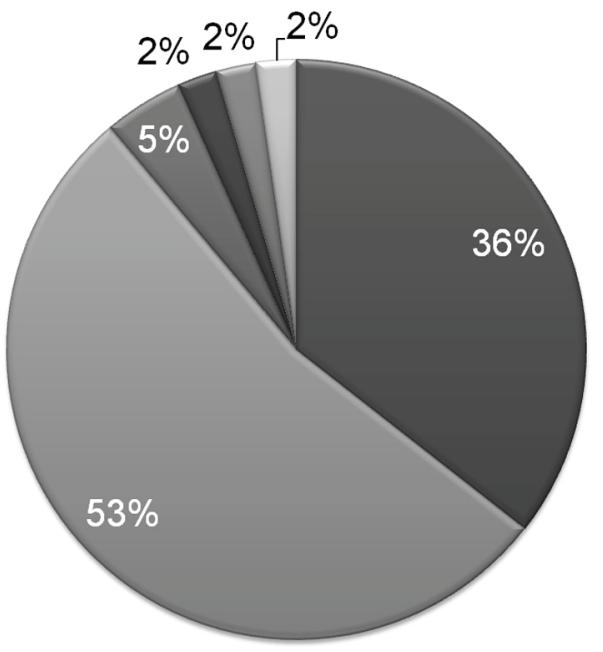

- Hypertrophic scalene muscle

口ibromuscular bands

$\square$ Broad first rib

Elongated transverse process $\mathrm{C7}$

$\square$ Large clavicule callus

$\triangle$ Narrow thoracic inlet

Figure 3. Intraoperative findings of an abnormal anatomy. 


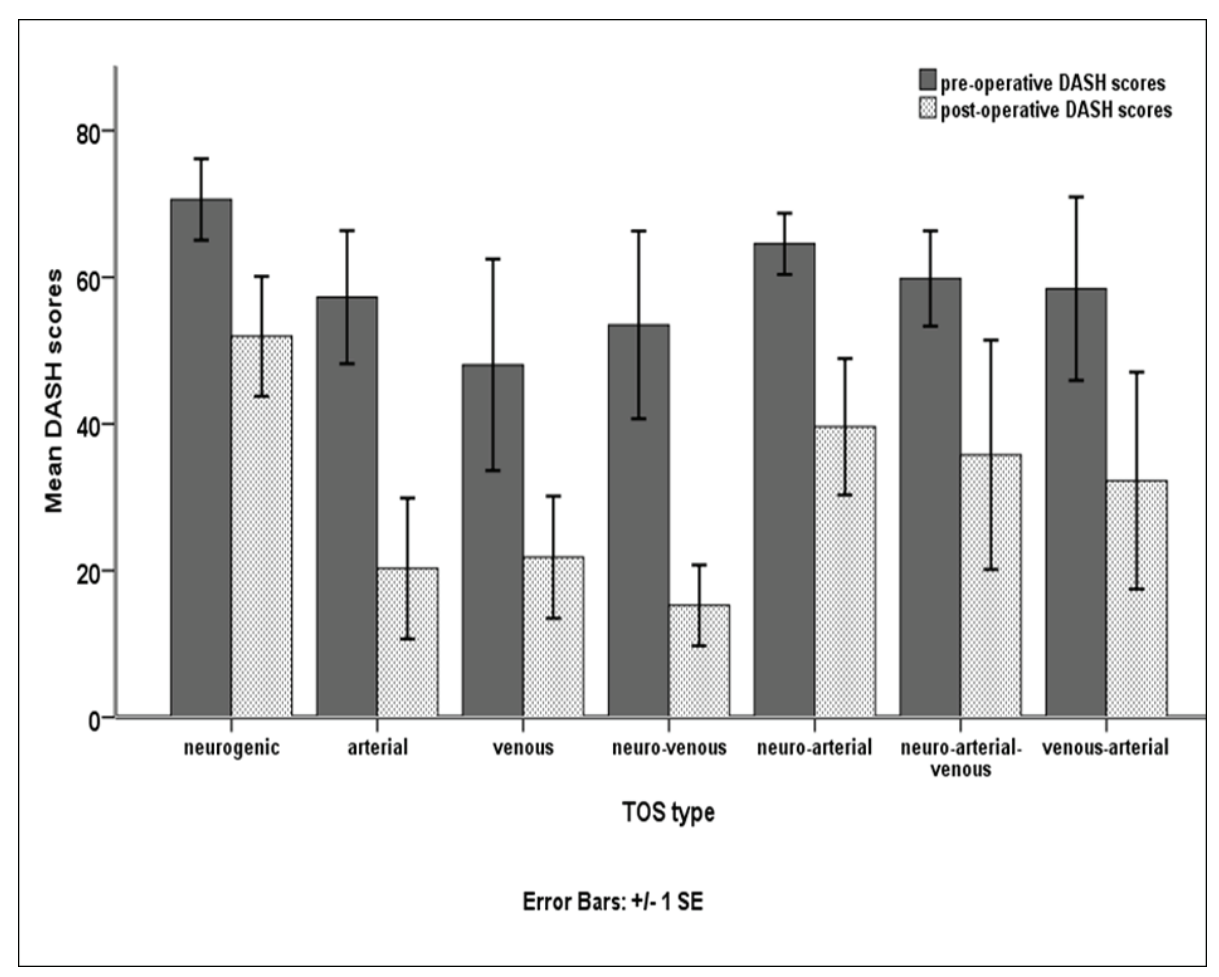

Figure 4. Pre and post-operative DASH scores.

naire comprises of four domains, which relate to the patient's symptoms, daily activities, social and psychological functions [7]. It contains 30 questions, where by scoring each item on the scale between 1 (no difficulty) to 5 (unable), patients describe the severity of their symptoms [7]. The overall score ranges from 0 points (asymptomatic patient) to 100 points (severe symptoms) [7, 13].

Statistical analysis was performed using SPSS version 13.0 software (SPSS Inc., Chicago). All data was observed to have a normal distribution and therefore parametric tests were used to compare variables. Within the group differences were assessed using paired t-test. Multiple comparisons were analysed using one-way ANOVA in conjunction with Tukey's method to identify true differences in means. Categorical data was analysed using the chi-square test. The data was assumed to be significant when $\mathrm{P}<0.05$.

\section{Results}

Of fifty patients, 44 (88\%) completed pre and post operative DASH questionnaires and these were included for further analysis. All results are presented as mean \pm SD unless stated otherwise. Mean age was $42 \pm 10.78$ years (range $16-60$ ) and 37 patients were women. Twenty seven patients had the combination of neurological and vascular symptoms. This group included 11 patients with nTOS, 8 with neuro-arterial TOS and 4 cases with neuro-venous subtype of TOS. The reminder of patients presented with vTOS $(\mathrm{n}=5)$, aTOS (n $=7)$ and 3 cases with venous-arterial TOS. All operations (40 elective and 4 emergencies) were performed under the general anaesthesia via supraclavicular approach by a single surgeon. The decompressive procedures consisted of an excision of the cervical rib $(n=11)$ or first rib $(n=23)$, combined cervical and first rib excision $(n=10)$, scalenectomy $(n=42)$ and division of soft tissue $(n=24)$. The operative findings revealed an abnormal anatomy, such as hypertrophic scalene muscles $(n=16)$, fibromuscular bands $(n=24)$, elongated C7 transverse process $(n=1)$, large clavicle callus $(\mathrm{n}=1)$, narrow thoracic inlet $(\mathrm{n}=1)$ and broad first rib $(\mathrm{n}=2)$ (Fig. 3), 16 presented with bilateral TOS and six of them have undergone bilateral TOS decompression. Post operative complications included wound infection $(n=1)$ and phrenic nerve traction injury $(\mathrm{n}=2)$. There were 12 pleural entries of which 3 required chest drains insertion and 7 cases had pleural drains inserted using the breached pleural window. In 2 cases pleura was repaired. There were no brachial plexus or vascular injuries. Six patients required revision procedures. Mean follow up was $11.7 \pm 15.4$ months. There were no statistically significant differences between the groups in pre $(\mathrm{P}=0.49)$ and post operative $(\mathrm{P}=0.104)$ DASH scores. However, when DASH scores were analysed according to the TOS type, the most marked post operative improvement was noted in aTOS with mean delta change of $36.99 \pm 22.72(\mathrm{P}=0.005)$, followed by vTOS $(26.22 \pm$ $16.89, \mathrm{P}=0.026)$ and $\mathrm{nTOS}(18.64 \pm 21.07, \mathrm{P}=0.015)$ (Fig. 
4). DASH questionnaire revealed return of upper limb function in $78 \%(\mathrm{n}=39)$ of the patients. Only 5 patients (nTOS) reported worsening of the limb function.

\section{Discussion}

As expected, nTOS was the most common in our series. The reminder of the cases had the combination of neurogenic and vascular symptoms. In such cases, the contribution of the vascular component is often regarded as negligible $[4,8]$. Although, all our cases had proven subclavian vessels involvement (stenosis or occlusion on arm abduction) on pre operative Duplex ultrasound scan, in principle they all were treated as nTOS. The recommended treatment involves a period of physiotherapy ( $4-6$ weeks) prior to any form of surgical decompression $[5,14,15]$. Physiotherapy manoeuvres such as postural exercises and the first rib mobilisation aim to reduce the pressure on the neurovascular structures within the thoracic outlet $[16,17]$. Other conservative measures include the anterior scalene block with the Botulinum toxin type A (BTA) or local anaesthetic injection under ultrasound, computed tomography or electromyography guidance $[5,18]$. Relief of symptoms could be viewed as a "pre operative" assessment whether the patients would benefit from the first rib resection with scalenectomy [5, 12]. However, at present including a randomised control trial there is no evidence to support the use of BTA injections to improve the function and reduce the pain in TOS [3, 19]. Therefore, in our department we do not perform anterior scalene blocks, because the data to support this is currently lacking. Instead, we provide an extended course of physiotherapy (3 months) following which repeated clinical evaluation with DASH is performed. Those patients who remain symptomatic after the course of physiotherapy are carefully counselled regarding the proposed surgical treatment.

The most common presentation of vTOS is an axillosubclavian vein thrombosis, which is also known as PagetSchroetter syndrome $[3,6,20]$. The objective of treatment is to prevent the recurrence of the thrombosis, which is associated with significant morbidity [21]. Treatment involves an early thrombolytic therapy (within 2 weeks) followed by an anticoagulation (1 - 3 months) and delayed surgery $[5,20]$. Due to logistic reasons we were unable to provide thrombolysis in our institution. Despite that all 5 cases of vTOS had successfully re-established veins patency with the 3 month course of oral anticoagulation followed by an elective delayed surgical decompression. There were no cases of re-thrombosis. However, due to small sample size these findings should be interpreted with caution.

The least common type is an aTOS [8]. Although patients usually present with varying degree of upper limb ischaemia, a retrograde embolisation causing stroke has also been reported $[6,22]$. The ischaemia can be caused by either an external compression of the SCA or emboli arising from the associated SCA aneurysm [22]. In our series, 7 patients suffered from aTOS and 4 of them presented on as emergencies. The treatment involved decompression of thoracic outlet with an arterial reconstruction with the autologous vein $(n=2)$. The reminder of patients (aTOS) presented also with the nTOS symptoms. Therefore, this group of patients was treated on semi-elective (more urgently) basis in order to avoid the development of an arterial damage. Some authors suggested treatment of aTOS with endovascular stent grafts [6]. However, the results were disappointing and demonstrated high rate of graft stenoses $[6,23]$. This reflects the underlying pathophysiology of TOS, where decompressive procedures, aimed to relive the extrinsic compression, play the key role in the treatment.

The surgical techniques used for TOS decompression include transaxillary, infra and supraclavicular approaches [24]. We have used supraclavicular approach, which in our opinion provides favourable access to all neurovascular structures [1]. Additionally, satisfactory exposure of the scalene muscles is possible as well as an arterial reconstruction when necessary. There were six revisions in our series and these included 2 cases where no scalenectomies were performed during the first surgery. This has prompted the change in our practice and since then scalenectomy is routinely performed in every case. Similar findings were reported by other authors who found disappointing results when no scalene muscles excision was done as part of the decompressive procedure [25]. In the remaining 4 cases, revision procedures involved: neurolysis of the adhesions around brachial plexus, excision of the scar tissue around SCA, division of pectoralis minor (via transaxillary root) and excision of an abnormal band from the latissimus dorsi (via transaxillary root).

Post operative complications are related to the main anatomical neurovascular structures within the thoracic outlet. These include pneumothorax, haemothorax, chylous leak, brachial plexopathy, Horner's syndrome, vascular and phrenic nerve injuries $[1,26]$. There were no vascular or brachial plexus injuries in our cohort of patients. 12 pleural entries were encountered, of which 2 were repaired with Prolene, 3 required chest drain insertion for a large pneumothorax, and 7 cases were treated with drains inserted through the apical pleural defect. There were no phrenic nerve injuries except 2 cases where traction injury occurred. Both these patients fully recovered and had radiographically confirmed resolution of the initially elevated diaphragm.

DASH scores revealed the greatest improvement in the aTOS group. This comes with no surprise, because aTOS is nearly always associated with an abnormal anatomy, which once corrected (bone or soft tissue excision), leads to alleviation of the symptoms. The literature reports success rate of more than $90 \%$ following surgery and in our series all patients with aTOS remain asymptomatic [22]. Successful 
outcome in vTOS is related to the vein patency rates [5]. Therefore, some authors accept the lack of changes in the post operative DASH scores, suggesting no episodes of rethrombosis as the most important clinical outcome [5]. We have demonstrated an improvement in the DASH scores with no recurrences of vTOS group, but the small sample size makes it impossible to draw the definitive conclusions.

The most encouraging are the nTOS outcomes, which showed statistically significant improvement in mean post operative DASH scores. It has been shown before, that careful pre-operative assessment of patients plays a key role in successful both functional and surgical outcomes [27]. However, within this group, 5 patients reported worsening of symptoms after surgery (worse post operative DASH score). All cases were complex, where orthopaedic and neurosurgical consultations were obtained. This may reflect the transient response in the nTOS where initial success rate of $93 \%$ is reported, but decreases with time to $64-71 \%[9,28]$. This group of patients received the longest follow up and received multiple courses of physiotherapy, which was temporarily beneficial in some cases.

\section{Conclusions}

The diagnosis and management of TOS can be challenging, and requires an understanding of the complex pathophysiology of the different subtypes. We have demonstrated that careful selection of patients with meticulous and adequate surgical technique can produce excellent results in all TOS variants.

\section{Financial Disclosures}

None.

\section{References}

1. Cronenwett JL, Johnston W, Consult E. Rutherford's Vascular Surgery, 2-Volume Set E-Book: Saunders 2010.

2. Roos DB. Thoracic outlet syndrome is underdiagnosed. Muscle Nerve. 1999;22(1):126-129; discussion 137128.

3. Sanders RJ. Vascular thoracic outlet syndrome. Eur J Cardiothorac Surg. 2007;31(4):753; author reply 753754.

4. Roos DB. Congenital anomalies associated with thoracic outlet syndrome. Anatomy, symptoms, diagnosis, and treatment. Am J Surg. 1976;132(6):771-778.

5. Fugate MW, Rotellini-Coltvet L, Freischlag JA. Current management of thoracic outlet syndrome. Curr Treat Options Cardiovasc Med. 2009;11(2):176-183.

6. Davidovic LB, Kostic DM, Jakovljevic NS, Kuzma- novic IL, Simic TM. Vascular thoracic outlet syndrome. World J Surg. 2003;27(5):545-550.

7. Cordobes-Gual J, Lozano-Vilardell P, TorreguitartMirada N, Lara-Hernandez R, Riera-Vazquez R, JuliaMontoya J. Prospective study of the functional recovery after surgery for thoracic outlet syndrome. Eur J Vasc Endovasc Surg. 2008;35(1):79-83.

8. Jamieson WG, Chinnick B. Thoracic outlet syndrome: fact or fancy? A review of 409 consecutive patients who underwent operation. Can J Surg. 1996;39(4):321-326.

9. Sanders RJ. Results of the surgical treatment for thoracic outlet syndrome. Semin Thorac Cardiovasc Surg. 1996;8(2):221-228.

10. Brantigan CO, Roos DB. Diagnosing thoracic outlet syndrome. Hand Clin. 2004;20(1):27-36.

11. Roos DB. Essentials and safeguards of surgery for thoracic outlet syndrome. Angiology. 1981;32(3):187-193.

12. Sanders RJ, Hammond SL. Management of cervical ribs and anomalous first ribs causing neurogenic thoracic outlet syndrome. J Vasc Surg. 2002;36(1):51-56.

13. Chang DC, Rotellini-Coltvet LA, Mukherjee D, De Leon R, Freischlag JA. Surgical intervention for thoracic outlet syndrome improves patient's quality of life. J Vasc Surg. 2009;49(3):630-635; discussion 635-637.

14. Hooper TL, Denton J, McGalliard MK, Brismee JM, Sizer PS, Jr. Thoracic outlet syndrome: a controversial clinical condition. Part 2: non-surgical and surgical management. J Man Manip Ther. 2010;18(3):132-138.

15. Brantigan CO, Roos DB. Etiology of neurogenic thoracic outlet syndrome. Hand Clin. 2004;20(1):17-22.

16. Brantigan CO, Johnston RJ, Roos DB. Appendix: use of multidetector $\mathrm{CT}$ and three-dimensional reconstructions in thoracic outlet syndrome: a preliminary report. Hand Clin. 2004;20(1):123-126, viii.

17. Novak CB. Conservative management of thoracic outlet syndrome. Semin Thorac Cardiovasc Surg. 1996;8(2):201-207.

18. Danielson K, Odderson IR. Botulinum toxin type A improves blood flow in vascular thoracic outlet syndrome. Am J Phys Med Rehabil. 2008;87(11):956-959.

19. Finlayson HC, O'Connor RJ, Brasher PM, Travlos A. Botulinum toxin injection for management of thoracic outlet syndrome: a double-blind, randomized, controlled trial. Pain. 2011;152(9):2023-2028.

20. Thompson JF, Winterborn RJ, Bays S, White H, Kinsella DC, Watkinson AF. Venous thoracic outlet compression and the Paget-Schroetter syndrome: a review and recommendations for management. Cardiovasc Intervent Radiol. 2011;34(5):903-910.

21. Hood DB, Kuehne J, Yellin AE, Weaver FA. Vascular complications of thoracic outlet syndrome. Am Surg. 1997;63(10):913-917.

22. Davidovic LB, Koncar IB, Pejkic SD, Kuzmanovic IB. Arterial complications of thoracic outlet syndrome. Am 
Surg. 2009;75(3):235-239.

23. Szeimies U, Kueffer G, Stoeckelhuber B, Steckmeier B. Successful exclusion of subclavian aneurysms with covered nitinol stents. Cardiovasc Intervent Radiol. 1998;21(3):246-249.

24. Sanders RJ, Pearce WH. The treatment of thoracic outlet syndrome: a comparison of different operations. J Vasc Surg. 1989;10(6):626-634.

25. Thompson JF, Webster JH. First rib resection for vascular complications of thoracic outlet syndrome. Br J Surg. 1990;77(5):555-557.
26. Gelabert HA, Jimenez JC, Davis GR, Derubertis BG, O'Connell JB, Rigberg DA. Early postoperative hemorrhage after first rib resection for vascular thoracic outlet syndrome. Ann Vasc Surg. 2011;25(5):624-629.

27. Hooper TL, Denton J, McGalliard MK, Brismee JM, Sizer PS, Jr. Thoracic outlet syndrome: a controversial clinical condition. Part 1: anatomy, and clinical examination/diagnosis. J Man Manip Ther. 2010;18(2):74-83.

28. Axelrod DA, Proctor MC, Geisser ME, Roth RS, Greenfield LJ. Outcomes after surgery for thoracic outlet syndrome. J Vasc Surg. 2001;33(6):1220-1225. 